\title{
MAGNETIC SHIELDING OF NEUTRAL BEAM SOURCES IN MIRROR FUSION TEST FACILITY
}

J. W. Shearer, T. A. Cutler, L. W. Dilgard, J. Fabyan,

P. M. Holl, G. D. Porter, and J. S. Colonias

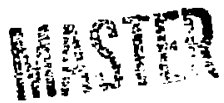

August 15, 1979

Work performeo under the auspices of the U.S. Department of

Energy by the UCLLL under contract number W-7405-ENG-48.
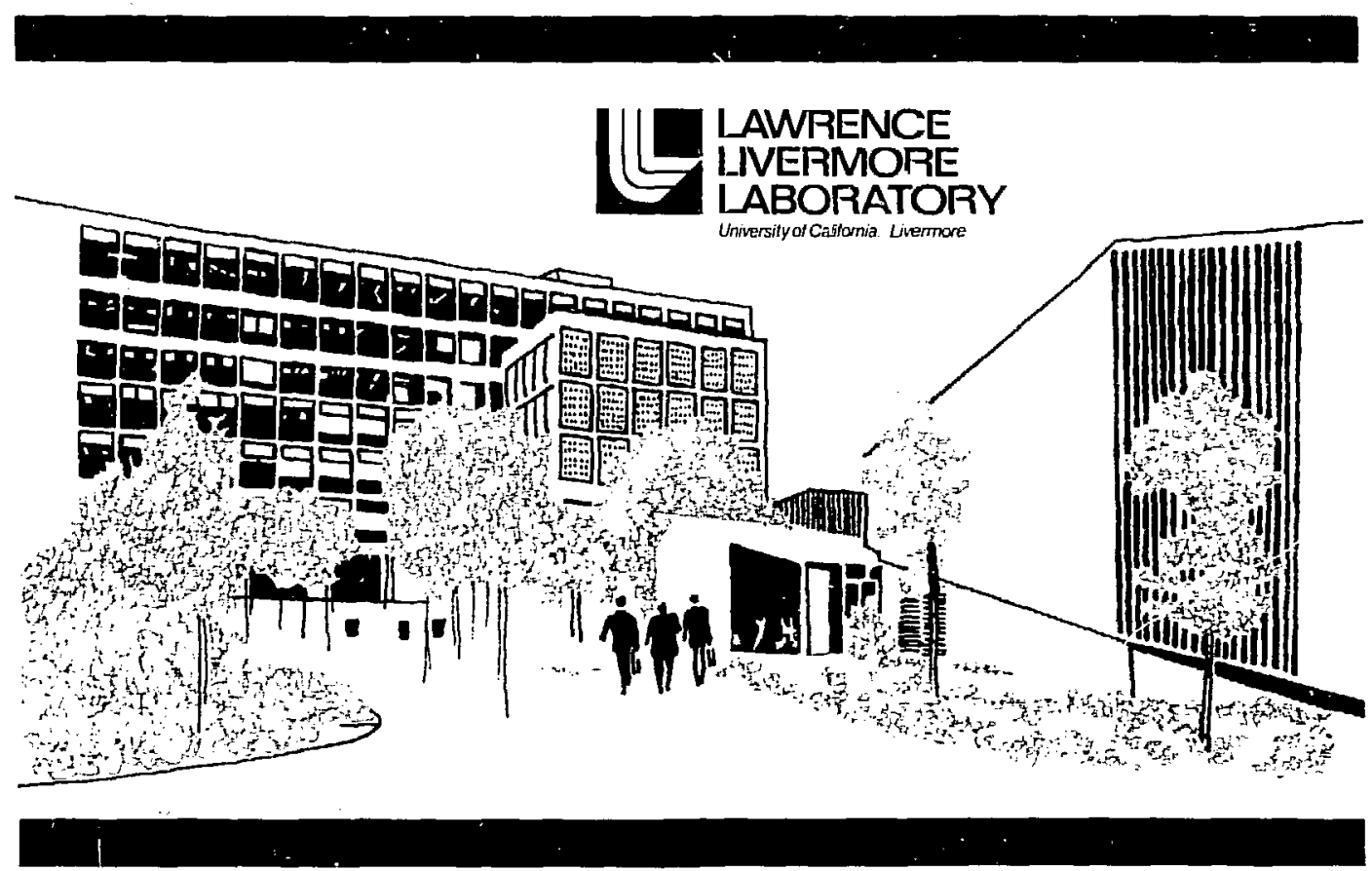


\section{近 \\ IAWRENCE LIVERMORE LABORATORY \\ University of Calfornia Livermore, California 94550}

UCAL-52824

\section{MAGNETIC SHIELDING OF NEUTRAL BEAM SOURCES IN MIRROR FUSION TEST FACILITY}

J. W. Shearer, T. A. Cutler, L. W. Dilgard, J. Fabyan,

P. M. Holl, G. D. Porter, and J. S. Colonias*

Manuscript date: August 15, 1979

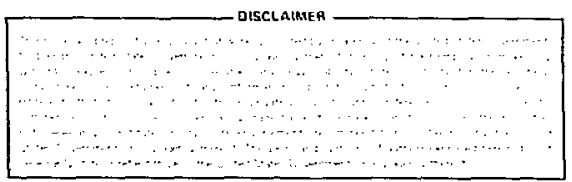




\section{CONTENTS}

Abstract ...................... 1

Introduction ..................... . . l

Three-Dimensional Magnetic Field Calculations With

Magnetic Materials Included (GFUN Code). . . . . . . 5

Two-Dimensional Magnetic Shield Calculations . . . . . . 7

Design of 1/10th-Scale Mockup Experiment . . . . . . 12

External Field Between 1/loth-Scale Modules in

Cartesian Geometry Array . . . . . . . . . . . . . 16

Shielded Field Mcasurements: Hysteresis . . . . . . 17

Measurements of Layered Shields... . . . . . . . 19

Measarements Along Neutral Beam Axis . . . . . . . 20

Measurements Near Back Plate .............. 21

1/10th-Scale Module Prototype Tests . . . . . . . . 22

Full-Scale Modules . . . . . . . . . . . . 27

Concluding Remarks ................... 28

Acknow ledgments . . . . . . . . . . . . . . 28

References ..................... 29 


\section{ABSTRACT}

The nagnetic shields of the Mirror Fusion Test Facility neutral beam sources must keep the inner magnetic field below $0.15 \mathrm{mT}$ inside an unsymmetrical ambient manetic field of 0.05 to $1.5 \mathrm{~T}$. Numerical computations and l/loth-scale experiments were used to arrive at a three-layer shiels design consisting of two thin nickel-iron alloy shells inside a thick mild steel shell. A $1 / 10 t h-s c a l e$ prototype test confirmed the final design.

\section{INTRODUCTION}

We will describe computations and measurements of the design of the magnetic shield for the neutral beam injection mndules of the Mirror Fusion Test Facility (MFTF). The overall MFTF experiment ${ }^{1}$ is intended to bridge the gap between present-day small mirror experiments and future mirror-type Eusion reactors. Its larger size and technical complexity impose more stringent design requirements in many facets of the design. The magnetic shield design problem is a particularly challenging example.

Figures 1 and 2 are two views of the central parts of the MFTF experiment. Forty-four neutral beam modules are attached to the walls of the vacuum chamber in four locations: top, bottom, north side, and south side. At each of these locations there are 16 available positions. The positions are tightly packed because of geometrical limitations. ${ }^{2}$ First, the beams must be aimed so that they do not strike the sides of the two yin-yang magnet cases. Second, they must not strike the modules on the opposite side. Third, they must intersect the plasma in a useful place. In addition, the neutral beam modules must be close to the plasma (approximately $6 \mathrm{~m}$ ) so that a tight focus can be maintained at the plasma for flexibility in experimentation.

Figure 3 pictures the main features of the neutral beam module, including the magnetic shield. The shield is also the vacuum chamber wall of the module. Thus the back plate must have vacuum-tight ports for the electrical leads, and the front end must be open to let the neutral beam emerge into the main MFTF vacuum chamber.

The fringing magnetic field of the cryogenic MFTF yin-yang magnet set is 2 to 3 orders of magnitude larger than the permissible magnetic field 


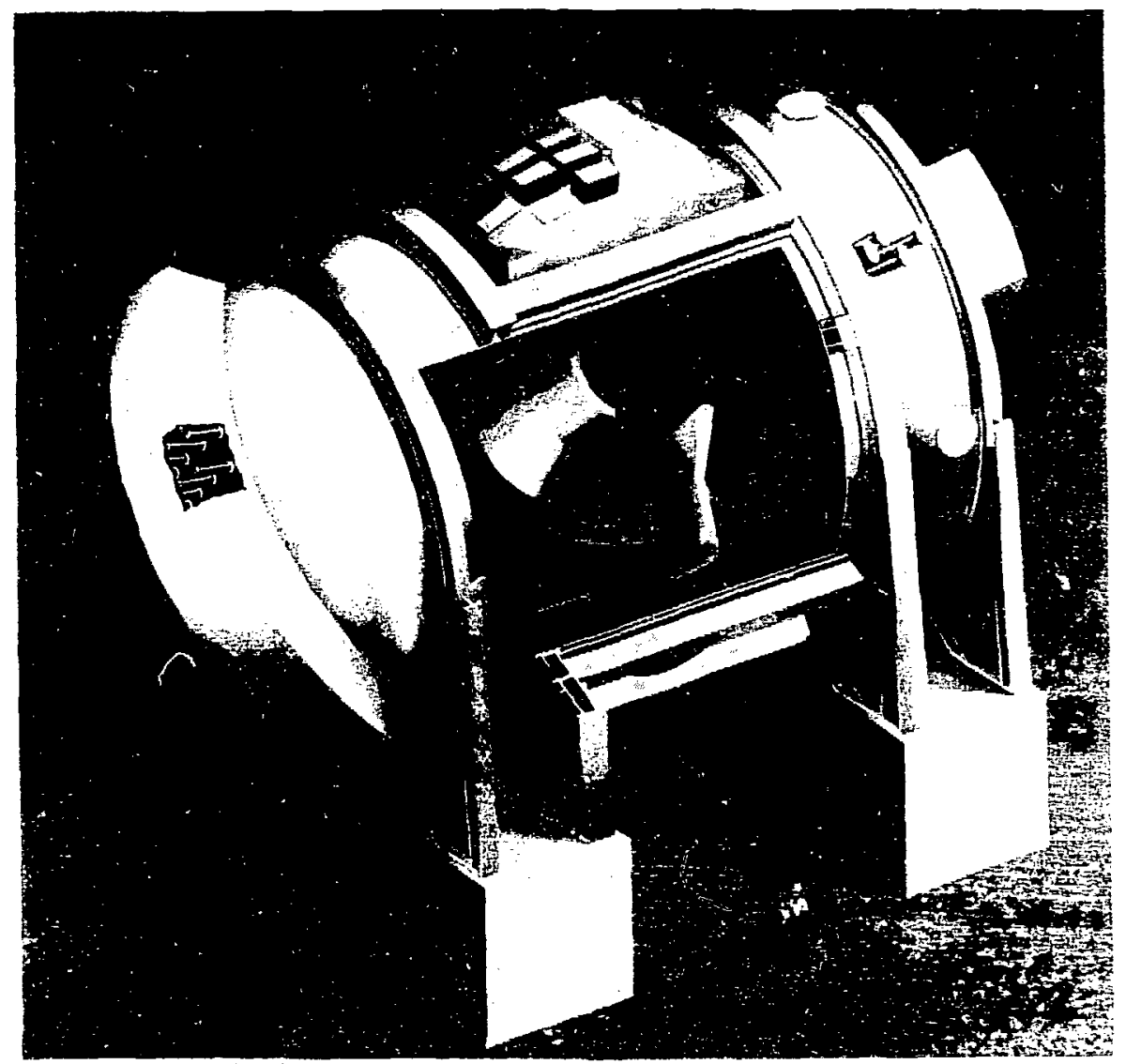

Fig. 1. Vacuurn vessel of MFTF, showing yin-yang magnets inside (array of 12 of neutral beam modules is on top of vessel, this being one of four pod lacations). Note restricted opening in magnet set through which horizontally mounted beams (see Fig. 2) must be aimed. 


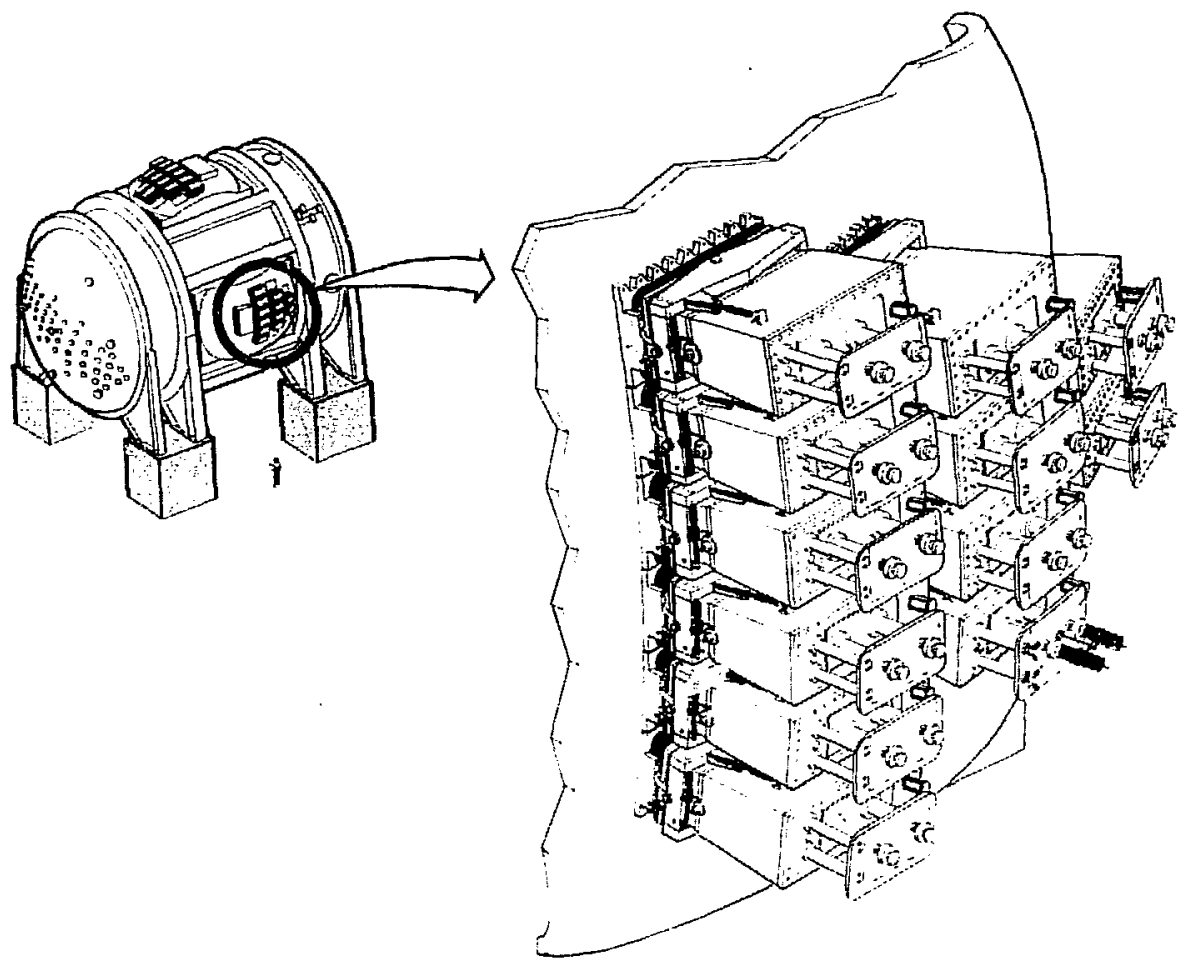

Fig. 2. Detail of array of 12 of 16 horizontally mounted beatas, showing high voltage feed-throughs mounted on back plates ef magnetic shielding boxes and aiming gimbals at wall of vacuum chamber. 


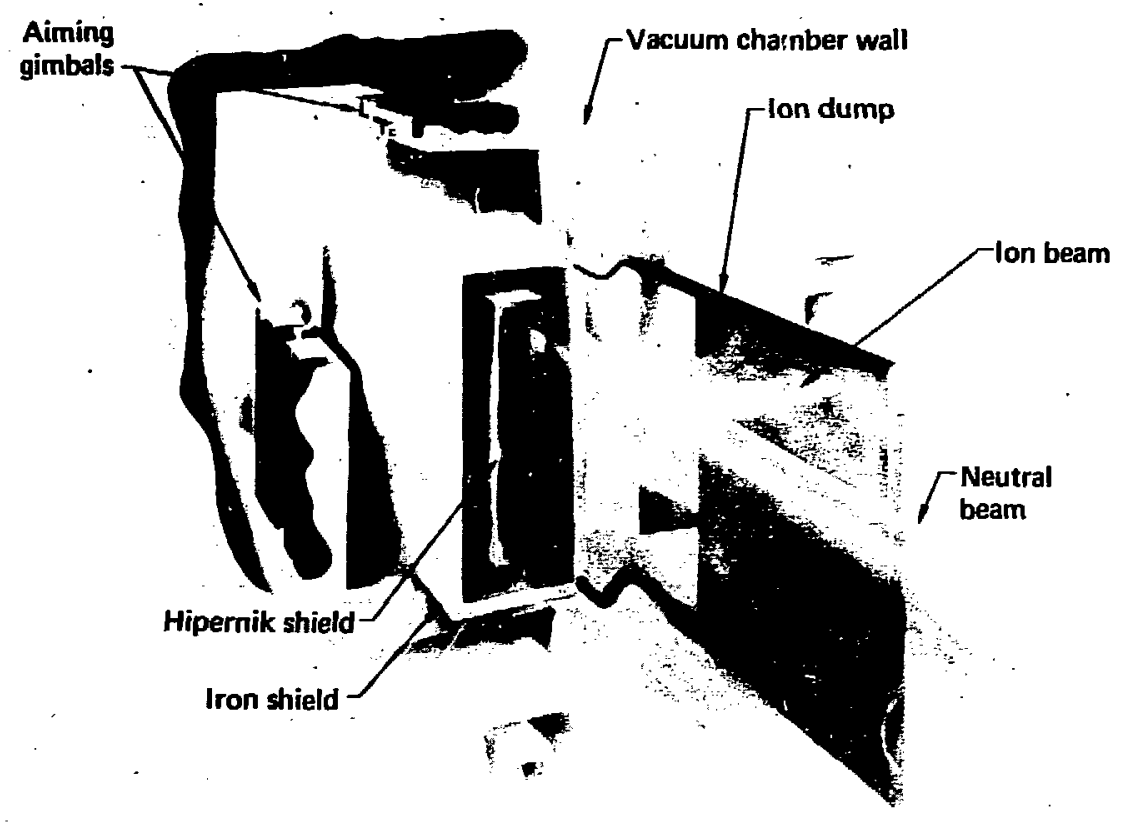

Fig. 3. Single module, showing beams emerging from magnetic shield--only one of two inner nickel-iron ("Hipernik") shields is shown here.

inside the neutral beam modules. In the arc chamber, an electron gyroradius condition imposes an upper limit to the magnetic field of $0.15 \mathrm{mT}^{3}$ On the other hand, the vacuum magnetic field in the region of the neutral beam modules is in the range of 30 to $100 \mathrm{mT} .^{4}$

Only the ferromagnetic properties of the iron are useful because, unlike $2 X I I B$ or $T M X$, the magnetic field is steady state, not pulsed. Space and weight constraints of the MFTF design limit the allowable total thickness of shielding to 50 to $75 \mathrm{~mm}$.

The direction of the vacuum magnetic field is somewhat variable over the neutral beam module array space (the "pod"), but is usually primarily transverse to the axis of the neutral beams. 4 However, as described later, the array of magnetic shields substantially alters the magnetic field environment of each individual shield. 
At first we atcempted to formulate the design using numerical techniques only. However, the uncertainties in these calculations caused us to design and perform some 1/10th-scale shielding experiments. In this report we describe only those calculations and experiments that influenced the final shielding design.

\section{THREE-DIMENSIONAL MAGNETIC FIELD CALCULATIONS} WITH MAGNETIC MATERIALS INCLUDED (GFUN CODE)

As is evident from Figs. 1 through 3, the general problem is threedimensional. The only available three-dimensional magnetic field code that also includes ferromagnetic materials is the GFUN code, originally developed at the Rutherford Laboratory in England. ${ }^{5}$ In principle, such a code should solve the whole magnetic shielding problem, but in practice there is a serious limitation--only 100 "elements" (zones) of magnetic material can be included in each calculation, and these elements should be approximately cubical for maximum accuracy. As is evident from Fig. 3, this number is far too few "elements" to represent accurately even a single shield, to say nothing of an array.

Nevertheless, useful results are obtained from this limited code. The entire MFTF coil geometry and one 16-module array of magnetic shields were mocked up in a three-dimensional GFUN calculation. Because of the zoning limitations the actual hollow shield geometry was replaced for each shield by a solid block of magnetic material. The magnetic permeability $\mu$ of this block was normalized so that the total magnetic moment was unchanged:

$$
\mu_{\text {block }}=\frac{V_{\text {shield }}}{V_{\text {block }}} \mu_{F e} \text {, }
$$

where $\mathrm{V}$ is the volume. Previous calculations on the ion dump trajectory problem ${ }^{6}$ had shown that this approximation is quite accurate for fields external to the shields.

The principal result of these calculations is displayed in Fig. 4, where the external field between selected modules is plotted vs distance from the center of the MFTF plasma. We find that the array of magnetic shields attracts flux lines from the inner radii and shifts them out to the 
shield region. The field is highest at large radius because of the trapezoidal shape of the shields; the air gaps are smallest near $7.0-m$ radius. The field directions have also changed; the lines cross almost directly across each gap, so that they minimize their path length in nonmagnetic space (air or vacuum), as one expects from the concept of magnetic reluctance.

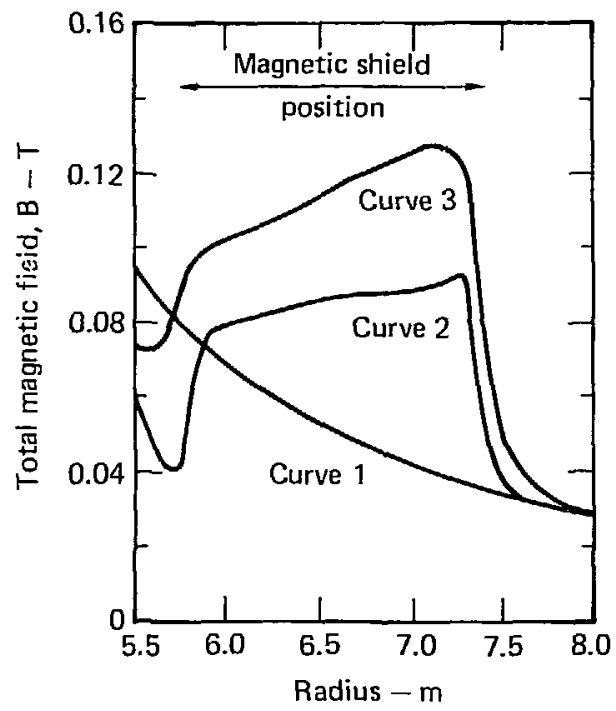

Fig. 4. GFUN code calculations of fringing MFTF magnetic field near neutral beam modules (Curve $I$ is vacuum field when no iron shields are present; Curve 2 is on a line midway between modules 613 and 614 , and Curve 3 is on similat line midway between modules 614 and 624 ; the radius is measured from center of MFTF plasma).

Figure 5 shows the extreme ranges in these calculations when all of the fields between modules are surveyed. The higher values are usually found near the outside of the array. In fact, the maximum fields are located along lines adjacent to module 611 , on one corner. Thus this module is the most difficult one to shield.

Fields surveyed immediately outside the module array a11 look like the vacuum field (Curve 1 of Fig. 4) with only minor differences.

The major lesson of these results is that the magnetic field environment of each module is fundamentally different in both magnitude and diIection from the vacuum field calculation. This difference is due to the effect of the other fifteen modules, and it must be taken into account in any further calculations. 


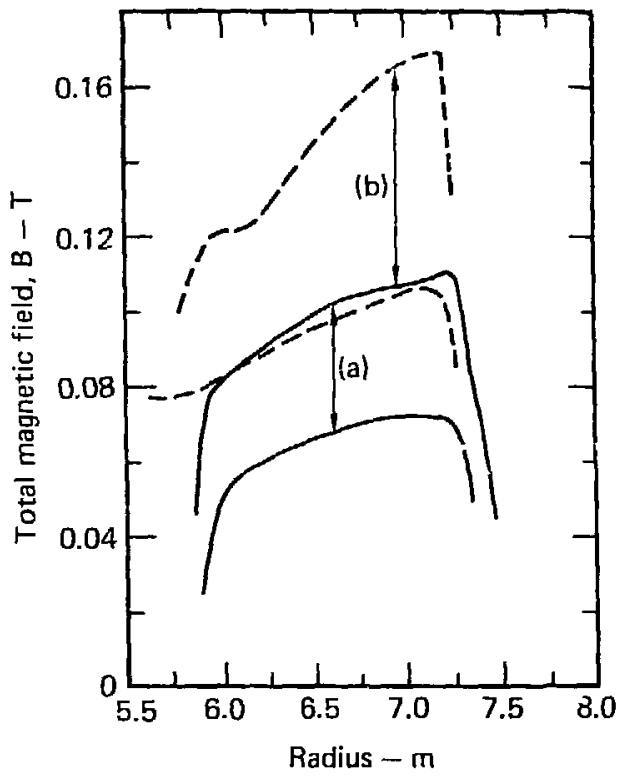

Fig. 5. More GFUN code calculations of fringing MFTF magnetic fields, showing extreme ranges for all lines between modules inside array: (a) is rarge for lines between adjacent modules in same tier (includes Curve 2 of Fig. 4); (b) is range for lines between tiers (includes Curve 3 of Fig. 4).

\section{TWO-:-:MENS IONAL MAGNETIC SHIELD CALCULATIONS}

More detailed computations of magnetic shielding are possible if only two spatial dimensions are involved. In order to do such calculations we model a full scale two-dimensional section of the magnetic shield at the arc chanber position, $7.0 \mathrm{~m}$ from the plasma center. Figure 6 shows the geometry of this section for problems involving three nested shields. We also calculate cases where one or both of the inner shields are omitted.

The TRIM code is used for these calculations. ${ }^{7}$ It employs a mesh composed of irregular triangles in which mesh lines are distorted to conform to the problem geometry. We use the de magnetization curves supplied by each manufacturer for the magnetic materials, as graphed in Fig. 7. Not shown here are some ineasurements of one actual sample of Allegheny-Ludlum 4750 alloy; both the measurements and a computer calculation based on them show little change from the corresponding parameters derived from the manufacturer's curve. 


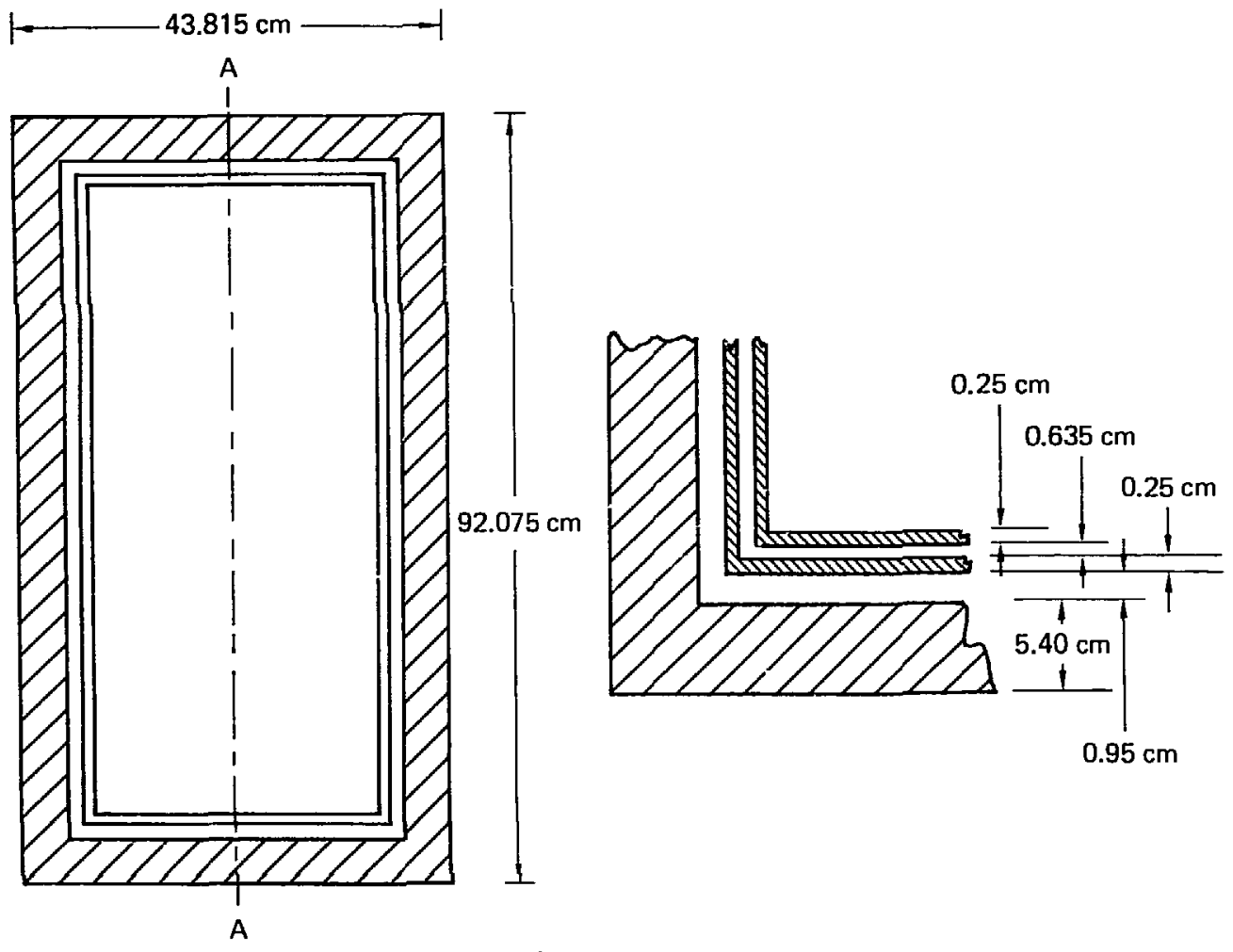

Fig. 6. Geometry used in two-dimensional full-scale TRIM calculations (a thick outer mild steel box is shown at left enclosing two thin inner shells of iron-nickel alloy; cross-section dimensions are given at right). 


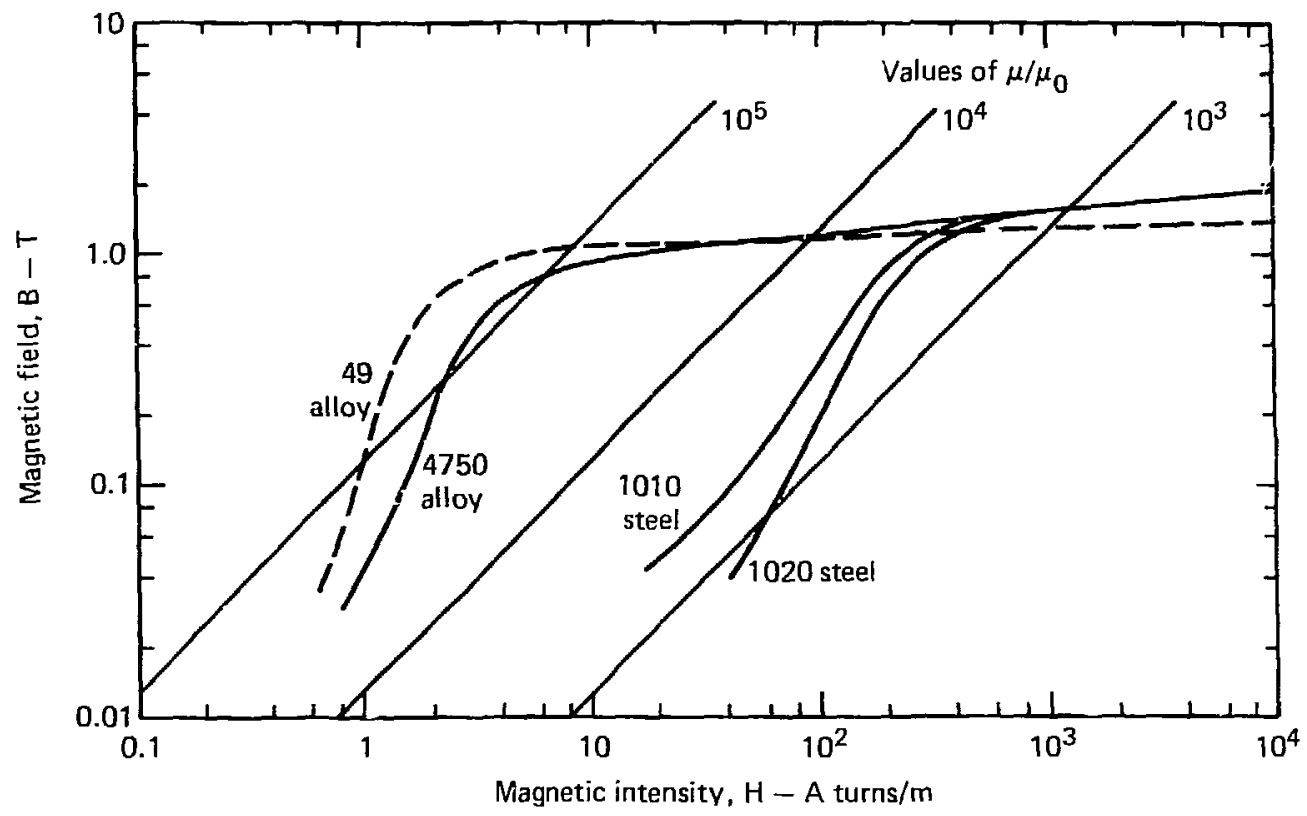

Fig. 7. Direct current magnetization curves for four magnetic irons, taken from industry data for AISI 1010 steel, AISI 1020 steel, Allegheny Ludlum 4750 alloy, and Carrenter High Permeability 49 alloy (first two are low carbon steels, and last two are iron-nickel alloys).

The code requires boundary conditions in the space outside the box. For these problems we adopt simple magnetic fields taken from the threedimensional GFUN code runs described above. These fields are 850 gauss along the long dimension and 1250 gauss along the short dimension of the box, corresponding closely to the results shown in Fig. 4. The field directions are normal to the box; closely matching the three-dimensional results. In order to test the sensitivity of the shielding to these boundary conditions, additional code runs are made with boundary "field multipliers" of 0.8 and 1.2 , roughly corresponding to the range seen for different modules. For a three-layer shield we find that the standard TRIM code mesh of 4,000 points is insufficient for adequate simulation. Both the inner layers and thr gaps nave to be at least three mesh lines wide to avoid convergence 
difficulties. Thus we find that a 10,000-point mesh is necessary to produce reasonable results.

In addition, a "zooming" technique is possible, where small sections of à coarse mesh are subsequently enlarged to achieve better results. This is also successful, but it requires too much time for data manipulation between the two meshes. Therefore, the 10,000-point mesh is used for the definitive calculations.

A selection of results from these full-scale shielding calculations is displayed in Figs. 8 and 9 . In Fig. 8 we show the effects of successive improvements of the shield design. Changing the soft steel from AISI.1020 to AISI 1010 reduces the fi.eld from 1.0 to $0.77 \mathrm{mT}$. Adding one inner shield of 4750 alloy then reduces the field to $0.38 \mathrm{mT}$. Finally, the complete three-layer shielding geometry brings the field dom to $0.055 \pm 0.005 \mathrm{mT}$, which is less than the 0.15-mT upper limit for the arc chamber.

Another calcu?ation, not shown here, uses one alloy shield at the inner positim, with the soft steel (AISI 1010) thickness increased from 5.4 to $6.6 \mathrm{~cm}$. It reduces the inner field just from 0.38 to $0.36 \mathrm{mT}$. Thus, a three-layer shield is more effective than a cwo-layer shieid within the same overall thickness range.

Figure 9 demonstrates the sensitivity of the shielding calculation to the value of the boundary field. Changing the magnitude of the outside field by $20 \%$ causes the inner, shielded field to change by a factor of 6 to 8. This extreme sensitivity arises from the saturation of one or more of the layers of the shield.

In summary, these calculations are both optimistic and pessimistic. In Fig. 8 the results pr'dict that a three-layer shield design is capable of achieving the required low field level at the arc chamber position. However, the results of Fig. 9 show that this prediction is subject to considerable uncertainty due to magnetic saturation effects. The previously described approximations necessary to set up these two-dimensional ca1culations of the three-dimensional box contribute additional uncertainty to the results. These uncertainties make it too risky to propose a final shielding design based on numerical calculations alone.

If there were a three-dimensional code with the detailed capabilities of TRIM, we would hope to be able to reach a shielding design by numerical calculation alone. Such a code is not presently available. 

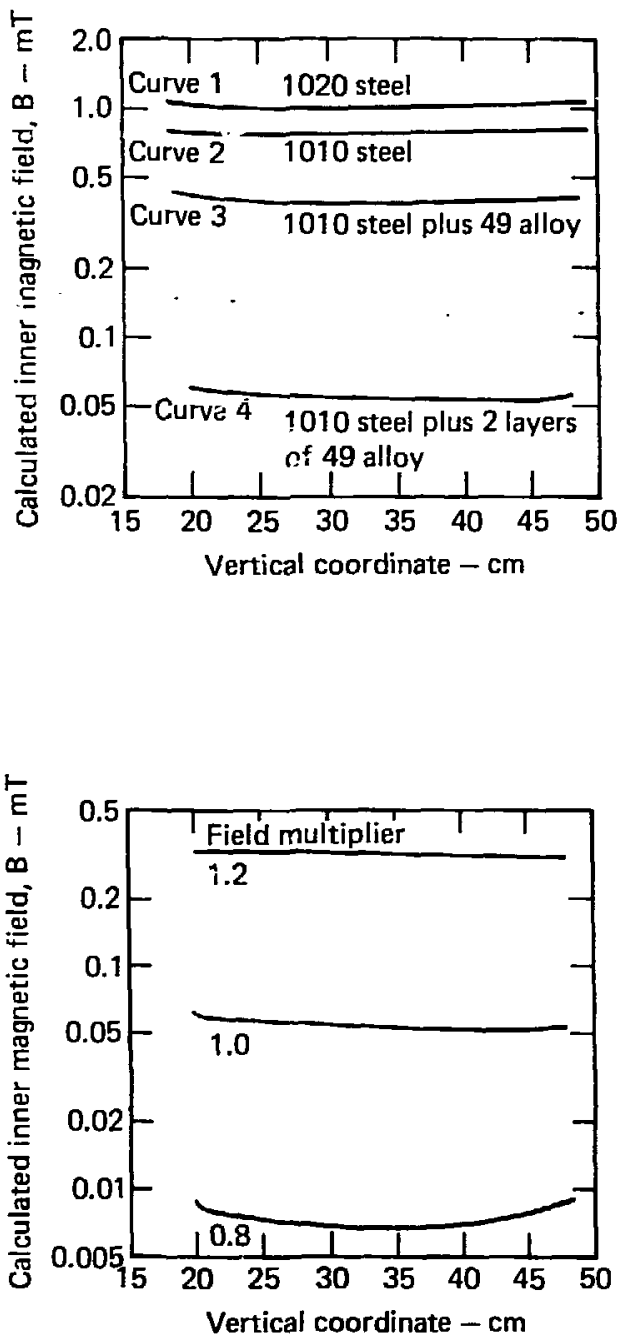

Fig. 8. Calculated inner magnetic field vs vertical coordinate along centerline AA of box shown in Fig. 6 (Curves 1 and 2 are without. any inner shields; Curve 3 uses on iy one inner shield; Curve 4 is For geometry of Fig. 6).

Fig. 9. Calculated inner magnetic field vs vertical coordinate for the case of two inner shields (Curve 4 of Fig. 8). Curves are labeled with "field multiplier"--a factor multiplied into external boundary condition magnetic fields. 
Although the calculations described above indicate that a three-layer shield is possible, the approximations of the codes contribute uncertainties to the results. The three-layer design needs additional supporting evidence. To meet this requirement we salculated and assembled a $1 / 10$ th-scale experiment to mock up the essential features of the module array.

The fundamental equation of magnetostatics that we scaled can be written:

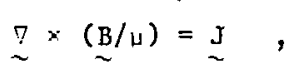

where $B$ is the magnetic field vector, $\mu$ is the local magnetic permeability, and $J$ is the local current density. To scale this equation geometrically we write:

$$
\underline{x}^{\prime}=\underset{\sim}{K x} \quad \stackrel{7}{\sim}^{\prime}=\frac{1}{K} \stackrel{7}{\sim}
$$

where $\underset{\sim}{ }$ is the displacement vector, and the scaled quantities are primed. We chose $\mathrm{K}=0.1$ for a $1 / 10$ th-scale experiment. In this case the gradients are all increasea by a factor of 10 , and thus the current density increases:

$$
J^{\prime}=\frac{\mathrm{J}}{\overline{\mathrm{K}}}=10 \mathrm{~J} .
$$

Because $B$ and $\mu$ remain unchanged, the scaled magnetic shields will have the same scaled magnetic field environment, provided the current density in the scaled coils is raised as shown in Eq. (4).

We did not have the time or the money to construct $1 / 10$ th-scale modules of the MFTF yin-yang coils--especially if their required current density had to be ten times that of the original coils. Instead, we approximated the $1 / 10$ th-scale MFTF vacuum fringing field by mears of two available coils. In the region of interest, where the shielding array is mounted, $\mathrm{J}=0$ everywhere. Thus, $\mathrm{B} / \mu$ can be linearly scaled provided that we can set up an initial magnetic field distribution that approximates the MFTF distribution (to 1/10th-scale).

The available coils had the following dimensions: i.d. $=406 \mathrm{~mm}$, o. d. $=711 \mathrm{~mm}$, and length $=156 \mathrm{~mm}$. The EFFI code ${ }^{8}$ was used to calculate the field distribution for various tilt angles of these two coils. The EFFI code is a three-dimensional magnetic field code (similar to the older MAFco code), which can calculate vacuum magnetic fields with considerable 
accuracy. It contains no magnetic materials, however. The $1 / 10$ th-scale experimental design was chosen by comparison of these vacum field calculations with the calculated vacuum field in the MFTF configuration.

After a few trials, the geometry of Fig. 10 was selected on the basis of the comparison of magnetic field profiles graphed in Fig. 11. For this comparison we used the MFTF vacuum magnetic field along the midplane between the neutral beam modules and the beam dump for the beams on the opposite side. (This field is slightly higher than the vacuum field used in Fig. 4.) The matchup is a compromise between accuracy and prudence; a closer agreement in slope could have been obtained by moving the shield mockups toward positive $Y$, but this move would have required a higher current in the water-cooled coils.

Calculations with these coils set at angles of 30 and $45 \mathrm{deg}$ were not as satisfactory a matci. as at $90 \mathrm{deg}$; the field gradient at the smaller angles was not steep enough in a practical current range. Furthermore, the variation of the field over a complete $3 \times 3$ array of $1 / 10$ th-scale model shields was too great. On the other hand, this variation was tolerable for the 90 deg coil setup (see Fig. 12).

The direction of the vacuum field in the MFTF neutral beam module array region was found to be primarily transverse to the radial line of sight of the neutral beams and about $45 \mathrm{deg} \pm 10 \mathrm{deg}$ with respect to the midplane. In the $1 / 10$ th-scale experiment, the direction of the field is primarily horizontal; therefore, the module array was rotated 45 deg to produce the desired orientation.

Figs. 13 and 14 show the actual experiment. A commercial three-axis Hall probe was used to probe the magnetic field; it was aligned by means of plastic tubing, which was attached to the frame at known positions. A scale was glued to the probe to measure position along the tube. Where necessary, corrections were made for the relative positions of the $B_{x}, B_{y}$, and $B_{z} d e-$ tectors.

The resistance of the two magnetic coils in series is of the order of $0.1 \Omega$. Thus, at $700 \mathrm{~A}$, about $50 \mathrm{~kW}$ of power must be provided, and the same amount of energy must be removed by water cooling. These requirements were met by locating the experiment in the east end of Building 436, where cooling water was available and where a large variable energy power supply could be borrowed. Most of the data reported here was taken with a $600-\mathrm{kW}, 0$ to 2000-A unit manufactured by PWR, Inc., destined for the BETA-II facility. 

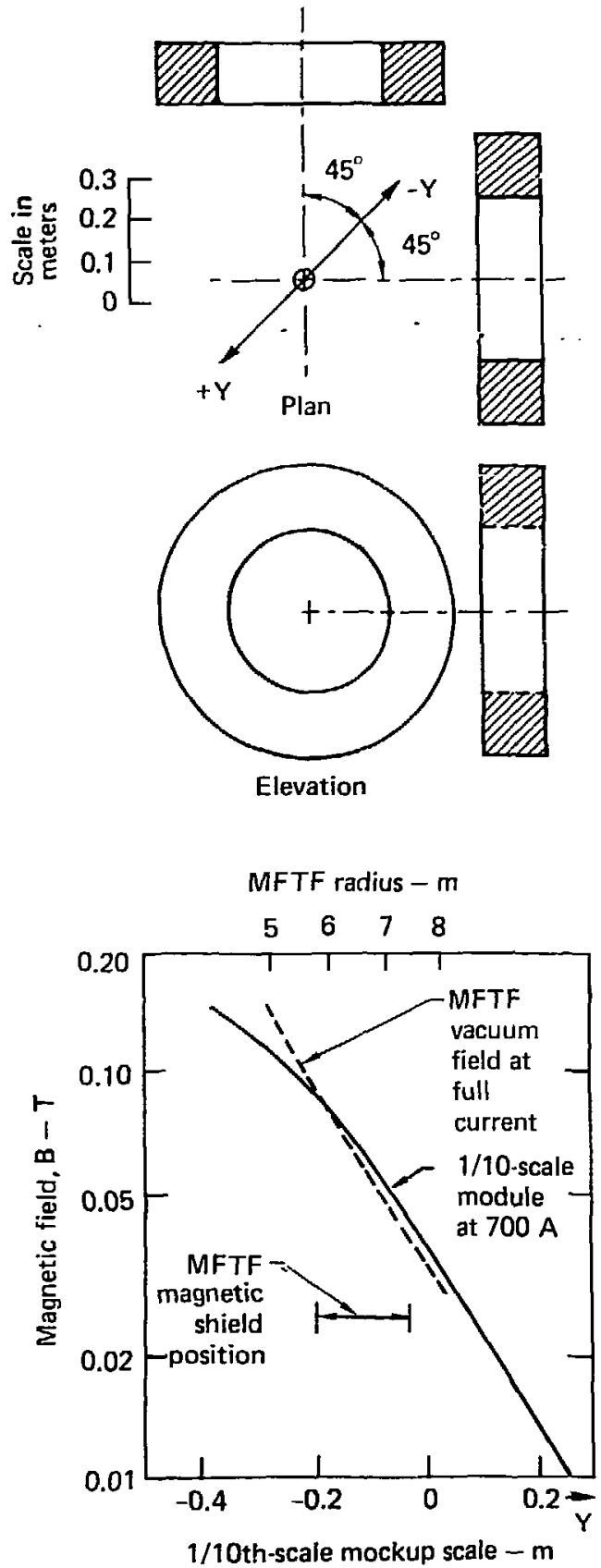

Fig. 10. Plan and elevation views of coil geometry used to approximate MFTF fringing field for 1/10th-scale magnetic shielding experiment.
Fig. 11. Matchup of MFTF vacuum magnetic field with vacuum field of 1/loth-scale experiment shown in Fig. 10. 


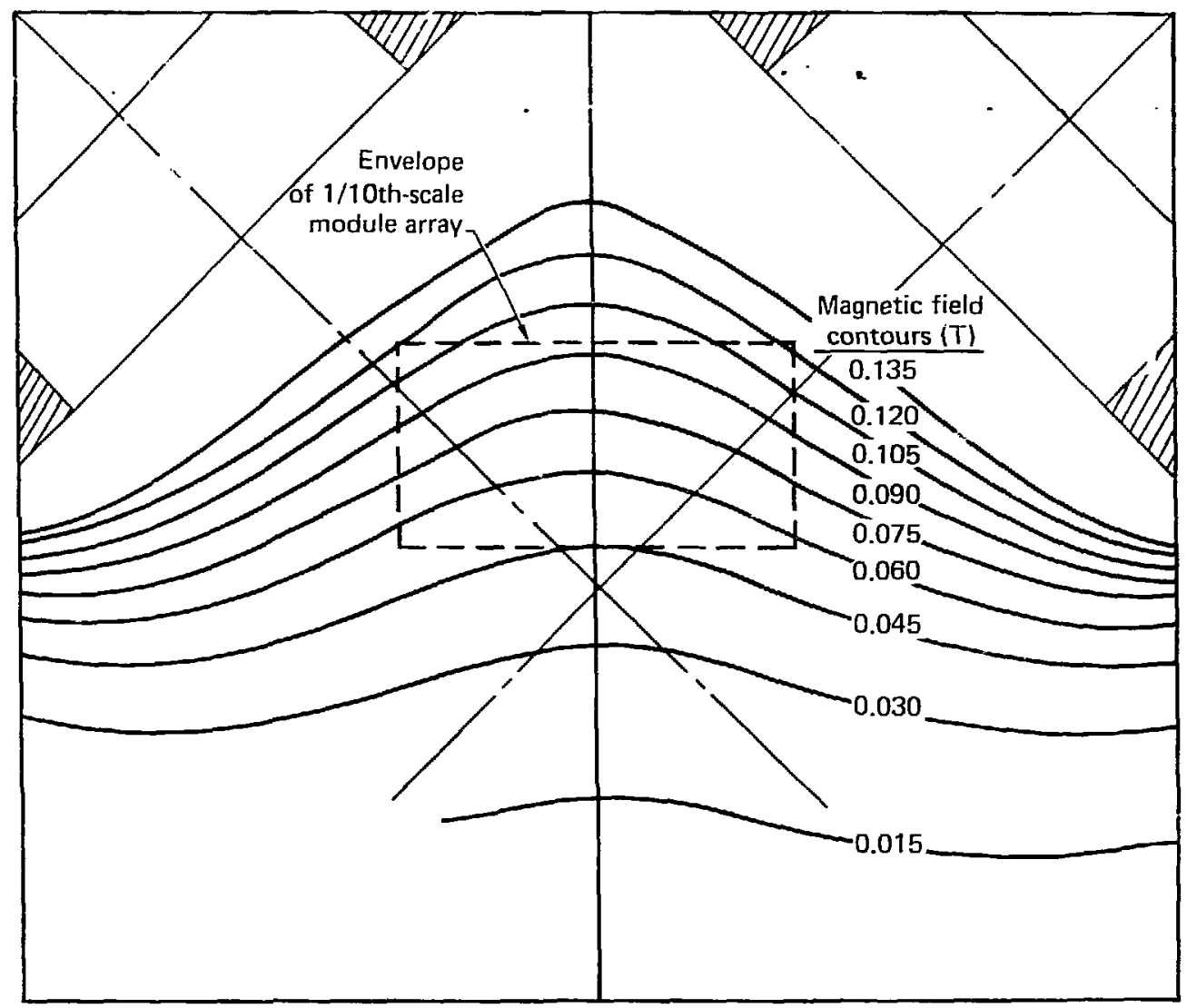

Fig. 12. Vacuum magnetic field contours in $X-Y$ plane of $1 / 10$ th-scale magnetic shielding experiment as calculated by EFFI code at $700 \mathrm{~A}$. 

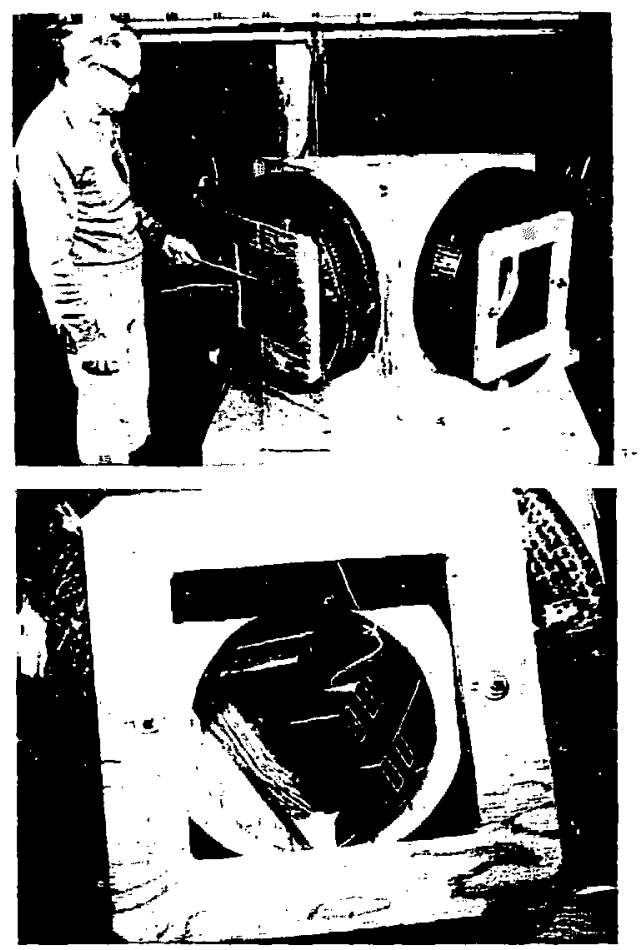

Fig. 13. Coil array for 1/10thscale experiment mounted in its supporting timber frame (note manually held Ha11 probe used in measurements).
Fig. 14. Coil axray as seen through left-hand coil of Fig. 13 (six shield mockups are seen, mounted at angle of $45 \mathrm{deg}$, as described in text; nine mockups were used in later tests).

Most of the exploratory data were taken with a Cartesian geometry array of $1 / 10$ th-scale model shields. The final prototype tests were made with a more exact "spherical" geometry ariay, which closely approximates the MFTF geometry.

EXTERNAL FIELD BETWEEN 1/10TH-SCALE MODULES IN CARTESIAN GEOMETRY ARRAY

Before studying the inside field, we took data along lines between shields in order to confirm the calculated deviations from the vacuum field. The GFUN code was used to calculate the geometry of the $1 / 10 \mathrm{th}-\mathrm{scale}$ experiment, and this calculation was compared to the data. Figure 15 displays this comparison for early data taken with an array of six "cowbells"--simple approximate 1/10th-scale shields made from available AISI 1020 steel. Experiment and calculation agree everywhere within $15 \%$. 


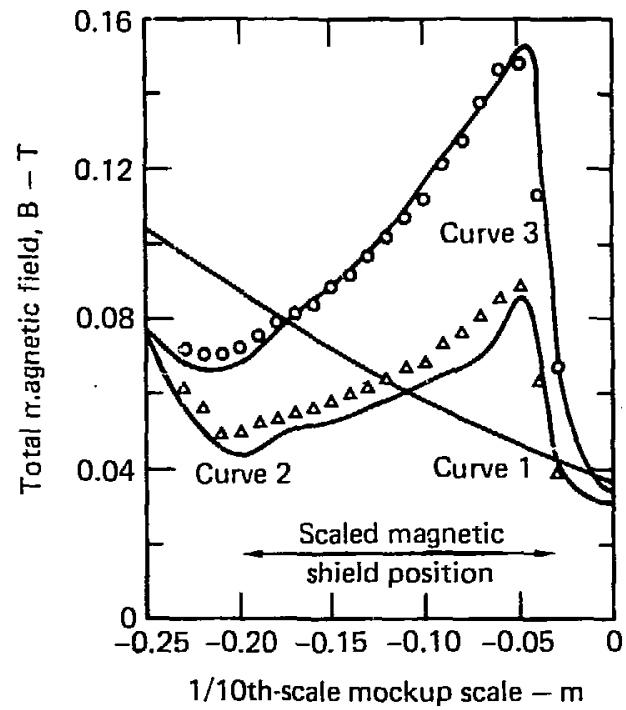

Fig. 15. GFUN code calculations compared with measurements of $1 / 10$ th-scale model for Cartesian (nonspherical) geometry array (Curves 1,2 , and 3 correspond to similar GFUN curves in Fig. 4; points are experimental data, and coil current was $700 \mathrm{~A}$ ).

The deviations from the vacuum field in Fig. 15 are similar to those calculated for the MFTF configuration of Fig. 4, but they are somewhat more extreme. This difference is due not only to the difference in slope of the vacuum field (see Fig. 11), but also to the Cartesian geometry of the l/10th-scale model array. Thus, the arc chamber ends were comparatively too close, and the neutralizer ends too far apart, compared to spherical geometry. Although this is only a qualitative matchup, it was sufficient for the exploratory measurements of parameter variations of the shield design. A more exact spherical array was used later for the prototype measurements.

We also found that the external magnetic field is almost a linear function of the coil current over the range $400 \leq \mathrm{I} \leq 700 \mathrm{~A}$, to an accuracy of $3 \%$, despite the nonlinearity of the magnetic permeability of the iron.

\section{SHIELDED FIELD MEASUREMENTS: HYSTERESIS}

We present selections from numerous measurements that best illustrate the important aspects of the magnetic shielding problem. We used an array of nine shields--one carefully machined and annealed scale model shield in the center, and eight cruder "cowbells" to mock up the environment of a typical MFTF module. Two different steels were studied for the thick outer 
shield: AISI. 1020 stee $1(\approx 0.2 \%$ carbon), and AISI 1010 stee 1 ( $\leq 0.10 \%$ carbon). The outer field distribution was the one shown in Fig. 15 for Cartesian geometry.

Some measurements of the inside field for these two thick shields are displayed in Fig. 16. The three-axis Hall probe was centered approximately 25 mm from the inside of the back plate, which corresponds to the scaled location of the arc chamber in the full-size module. As the current (and the corresponding outside field) is raised, the inner field rises slowly. At maximum current, the iron begins to saturate, and the inner field rises more rapidly. However, when the current is lowered, the inner field drops precipitously--even reaching the opposite sign over much of the range (because the field is predominantly transverse, polarity is definable even for the total field). This behavior is a consequence of hysteresis effects in the iron.

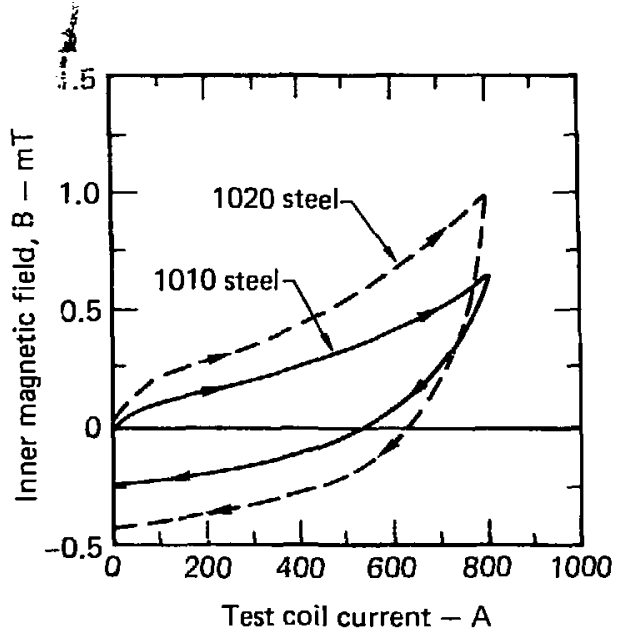

Fig. 16. Measurements of shielded field at arc chamber position for l/10th-scale experiment for two different steel shields plotted vs test coil current (arrows indicate sequencing of current supply; shape of curves is result of hysteresis effects--see text).

The hysteresis effect is due to the magnetization of the iron by the large external field. When that field is lowered, some of the magnetization remains "frozen-in." Inside the box the return flux from the remanent magnetization has the opposite sign from the external field; thus, it can efEectively cancel that field.

It is tempting to consider the possible application of this hysteresis effect to the MFTF shielding problem. The idea would be to raise the field above the desired operating point, and then bring it back down, so as to impress the desired magnetization in the shield. Unfortunately, the idea 
is not practical, because the MFTF superconducting magnets will be operated at their maximum design current. It is not possible to exceed that current without exceeding the critical field for the $\mathrm{Nb}-\mathrm{Ti}$ superconductor--thus forcing normal conductivity, and quenching the magnet.

Because we require the arc chamber field to be less than $0.15 \mathrm{mT}$ and because we must approach the maximum current ( $700 \mathrm{~A}$ in the scale model) from below, we find from Fig. 16 that neither AISI 1020 or AISI 1010 thick steel alone is sufficient for the magnetic shield. The AISI 1010 steel is obviously a superior steel for the application, however, and is being planned for MFTF.

\section{MEASUREMENTS OF LAYERED SHIELDS}

Thin layers of iron-nickel alloy provide additional shielding at low fields inside the thick stee1. For the $1 / 10$ th-scale model tests we used the alloy Carpenter 49, which was readily available in thin sheets. This material has much larger magnetic permeability than the stee 1 at low fields (see Fig. 7). The thin 49 alloy sheet was separately annealed and carefully mounted inside the steel with nonmagnetic spacers to provide $1 / 10$ of the gap spacings shown in Fig. 6. An ohmeter measurement confirmed that the two metal shells did not touch.

Figure 17, done in Cartesian geometry, shows the rising portion of the hysteresis curve (the "dc magnetization curve") for three cases: one 1010 steel layer; 1010 steel plus one 49 alloy layer; 1010 steel plus two 49 alloy layers. The results demonstrate that two inner alloy layers effectively reduce the inner field below $0.15 \mathrm{mT}$ over the entire current range measured--that is, un to $900 \mathrm{~A}$, which is we 11 above the $700-\mathrm{A}$ leve1 that matches MFTF (Figs. 11 and 15). One inner layer improves the performance of the steel but not enough to meet the criterion of $0.15 \mathrm{mT}$ at $700 \mathrm{~A}$. We believe that the first 49 alloy layer starts to saturate near $500 \mathrm{~A}$, accounting for the subsequent $r$ ise in inner field at higher currents. But the second 49 alloy layer doesn't begin to saturate until the current exceeds 800 A. 


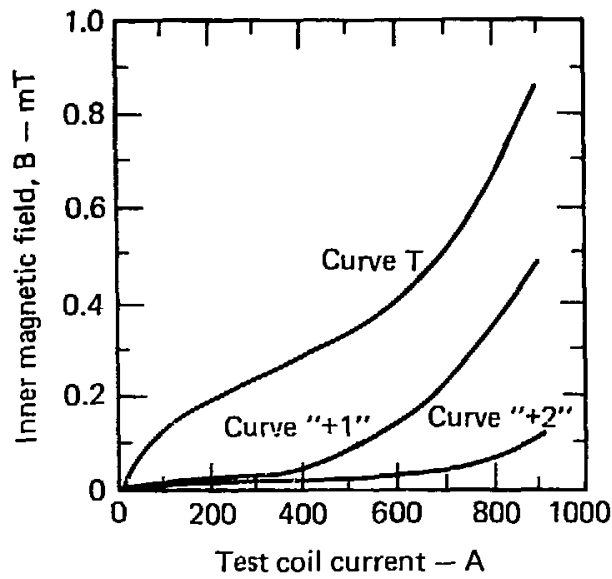

Fig. 17. Measurements similar to Fig. 16 for three cases: Curve $T=$ thick 1010 steel shield alone; Curve $" 1 "$ = same shield plus air gap plus $0.25 \mathrm{~mm}$ of 49 a11oy; Curve $"+2 "=$ shield plus two $0.25-\mathrm{mm}$ layers of 49 alloy.

This result demonstratas the necessity of using a three-1ayer magnetic shield for the MFTF neutral beam modules.

MEASUREMENTS ALONG NEUTRAL BEAM AXIS

We surveyed the magnetic field throughout the length of the $1 / 10$ thscale module mockup because of the additional criterion on the line integral of the magnetic field in the beam path (ion accelerator plus neutralizer) ${ }^{3}$ :

$$
\begin{aligned}
& \int \mathrm{Bd} \ell \leq 0.2 \mathrm{mT}-\mathrm{m} \quad \text { (MFTF) } \\
& \int \mathrm{B}^{\prime} \mathrm{d} \ell^{\prime} \leq 0.02 \mathrm{mT}-\mathrm{m} . \quad(1 / 10 \mathrm{th}-\mathrm{scal} l)
\end{aligned}
$$

Figure 18 shows measurements in Cartesian geometry for the recomended thick shield of AISI 1010 steel plus one or two inner layers of the 49 alloy. For the single inner layer shield, the above criterion is not quite met because the $1 / 10$ th-scale beam path will extend from -0.17 to $-0.20 \mathrm{~m}$ (approximate$1 y)$, and the corresponding area under Curve " $+1 "$ exceeds $0.02 \mathrm{mT}-\mathrm{m}$. The double inner layer shield, however, easily meets the same criterion. Thus, these results provide a second reason for needing the double layer shield. 


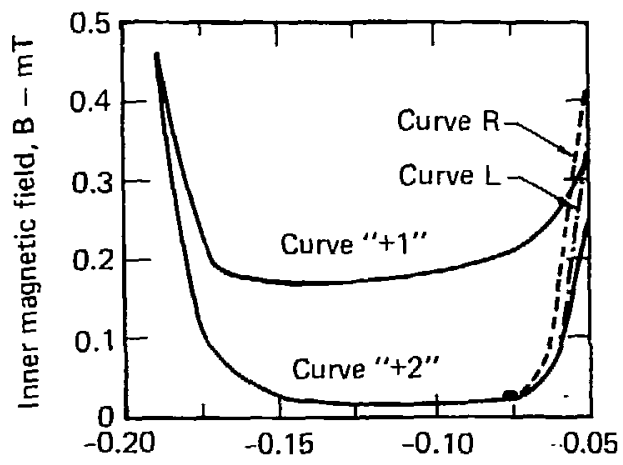

$1 / 10$ th-scale müikup scale $-m$
Fig. 18. Magnetic field scan along neutral beam axis at test coil current of $700 \mathrm{~A}$ (arc chamber position at $-0.07 \mathrm{~m}$ ). Curve $"+1 "=1010$ steel shield plus one 49 alloy layer; Curve "+2" = 1010 stee 1 shield plus two 49 alloy layers. Curves $R$ and $L$ are like Gurve " 2 " except scans are displaced 19 and . $13 \mathrm{~mm}$, reepectively, from neutral beam axis (1/10th-scaie module dimensions).

Note that the field rises at each end of the shield in Fig. 18. This rise is an obvious effect at the open end where the beam emerges and no shield at all is allowed. At the closed end there is only a single thick back plate made from AISI 1010 steel. The inner 49 alloy layers do not cover this back plate because of design and construction difficulties involved with the access holes and with the necessity to remove the back plate to change arc source filaments. Thus, some field lines can leak in from the back end also.

The arc chamber location for this model is at the edge of the fringing field from the back plate ( -0.07 m in 1/10th-scale model); it could not be located farther from the plate. ${ }^{8}$ However, this field is well below the $0.15-m T$ upper limit.

MEASUREMENTS NEAR BACK PLATE

There was considerable discussion about the effects of the back plate design, and many measurements were made related to this subject. Figure 19 is a Cartesian geometry comparison of the standard thick back plate with a thinner one, showing how the greater nonlinearity in the thinner plate raises the field everywhere, especially near tne back flate and at the access holes. At the arc charber position $(25 \mathrm{~mm}$ from the back plate in 1/10th-scale model), the thin back plate shield no longer satisfies the 0.15-mT upper limit criterion. Thus, we find that we must use the thick back plate despite its larger weight and greater handling problems. 


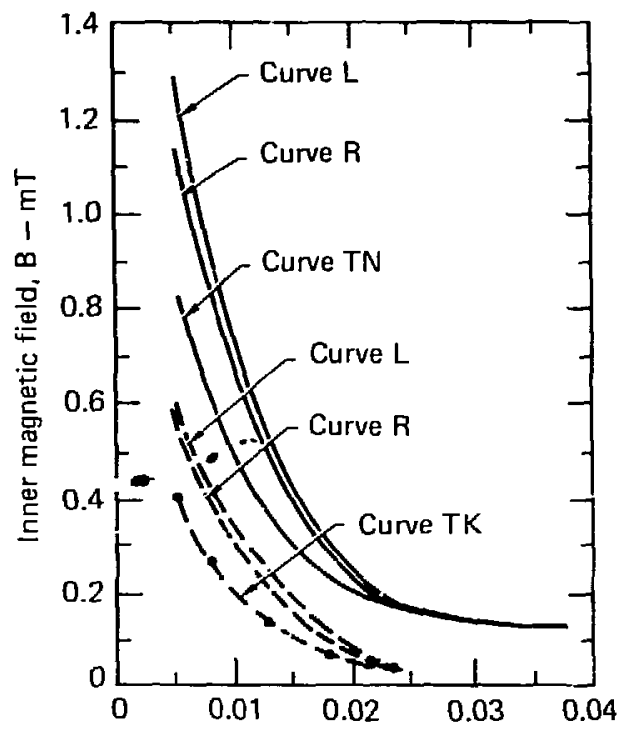

Fig. 19. Inner magnetic field vs distance from inside of back plate For 1020 steel plus two 49 alloy inner shields at $700 \mathrm{~A}$. Dashed lines are for standard thick (Curve $\mathrm{TK}=5.41 \mathrm{~mm}$ at $1 /$ 10th scale) back plate-using same scan lines--Curves $R$ and L-as for Fig. 18; solid lines are same measurements for thinner (Curve $T N=2.54 \mathrm{~mm}$ at $1 / 10$ th scale) back olate.

Distance from inside of back plate (1/10th-scale $)-m$

Other measurements, not presented here, were made of a number of details. Back plates without access holes were superior, but of course cannot be used. Heat-treated back plates were superior to those not heat-treated. Steel screws to attach the back plate are desirable for their magnetic properties.

\section{I/1OTH-SCALE MODULE PROTOTYPE TESTS}

The results of the calculations and measurements described thus far were incorporated into the formulation of the MFTF magnetic shield design. Additional changes were necessitated by the requirements of the $80-\mathrm{keV}$ sustaining beam module design. ${ }^{9}$ The final design is shown (approximately 1/10th-scale) in Fig. 20. The two end plates and the walls of the outer shield at the larger end are made of $0.54-\mathrm{cm}-$ thick (1/10th-scale) AISI 1010 steel. The outer shield walls at the smaller end (the neutralizer location) are $0.41-\mathrm{cm}$-thick (1/10th-scale) AISI 1010 steel. Various holes, some of which are shown in Fig. 20, are located so as to provide electrical and mechanica! access to the full-scale module. The inner shields are thin 

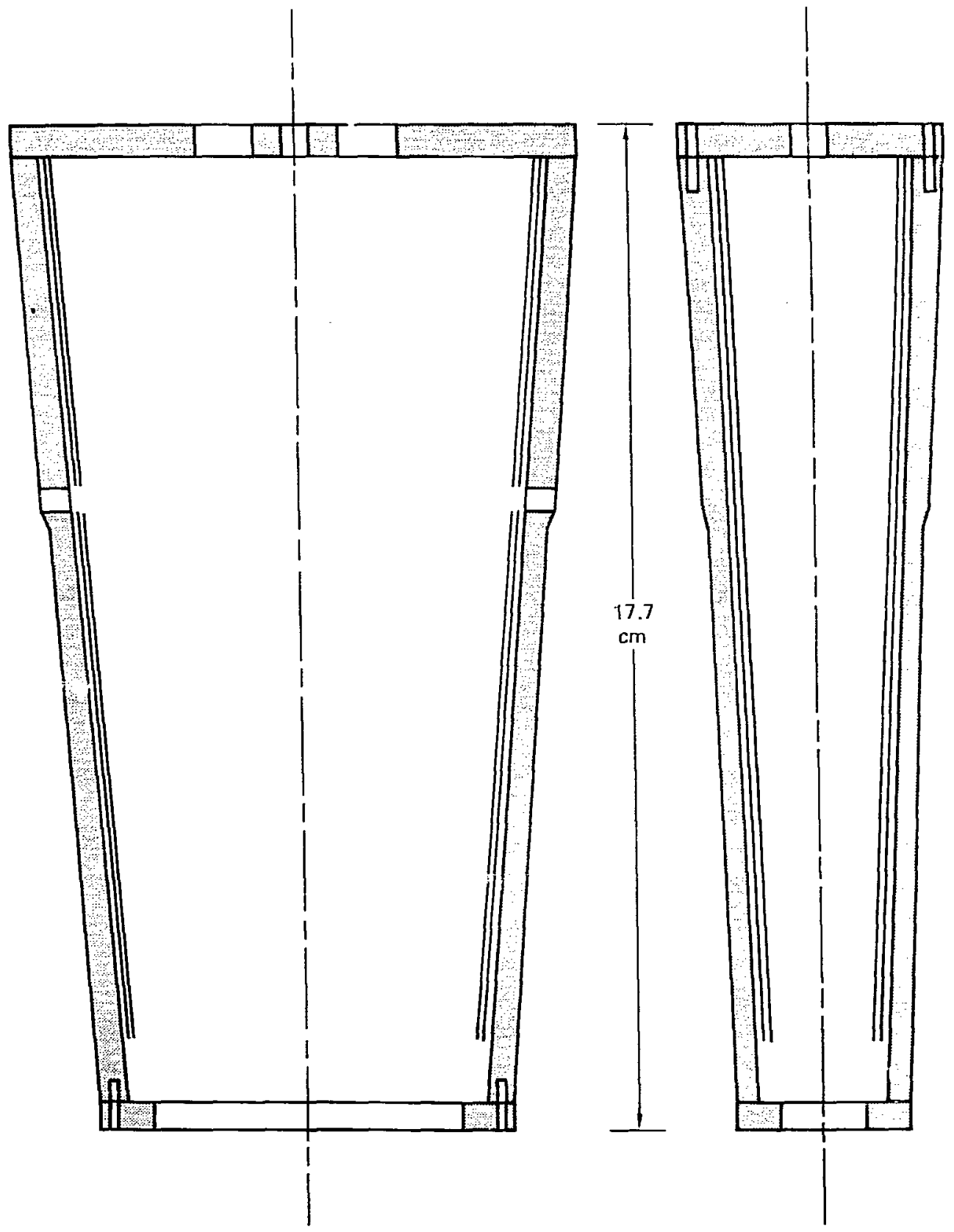

Fig. 20. Two sections of $1 / 10$ th-scale prototype shield approximately to scale (note front and back plates and change in thickness near the two gimbal mounting holes; thicknesses of inner shields and gaps are as shown in Fig. 6). 
$(0.025 \mathrm{~cm}$ in $1 / 10$ th scale) sheets of 49 alloy, assembled in the same way as previously described for the earlier inner shield measurements.

This prototype was mounted in a $3 \times 3$ array of $1 / 10$ th-scale modules as in previous tests. However, for these tests we used an improved mounting that mocks up the spherical geometry of the MFTF neutral beam pods (Fig. 2). Th-ee different arrays were tested, with the prototype mounted in the center, at one edge, and on one corner, respectively. Thus, we tested the shielding ability of the prototype under three different conditions representative of the different magnetic environments to be expected at different positions in the MFTF module arrays.

Figures 21 and 22 are a comparison of the measured total external magnetic field with the range of fields predicted by the GFUN code for MFTF (taken from Fig. 5). These measurements were made at $800 \mathrm{~A}$ in the test coils (instead of the 700 A previously used) in order to provide a more severe test of the prototype shield. In general, Figs. 21 and 22 show that these tests, especially the one at the edge of the array (Curve 2), are almost equivalent to the most severe magnetic field environment one expects to encounter in the MFTF experiment. The comparison is still not an exact cne, but is closer than that for the Cartesian array previously used, for which the field patterns (Fig. 15) w-re a poorer match to the predicted MFTF array fields.

It is interesting to note the effect of the "shoulder" in the shield thickness near the gimbal holes (Fig. 20). The external field increases outside the thicker part of the shield due to the narrowing of the gap between it and the neighboring shield.

Figure 23 plots the crucial results of the prototype test. It shows all of the measurements of the sinielded inside magnetic field plotted vs the distance from the back plate. In all three sets of measurements, the inner field is much less than the $0.15-m T$ upper limit for the arc chamber. The inner field is evidently somewhat higher $(\approx 0.05 \mathrm{mT})$ for the case in which the external fields are higher (Curve 2 in Figs. 21 and 22), but it is still we11 below the limit. The $0.02 \pm 0.01-\mathrm{mT}$ level of the other two cases is so low that the pexcent accuracy of the measurement is poor. Comparison with similar measurements in Cartesian geometry (see Fig. 18) shows no essential differences in the magnitude and shape of these curves. Thus, in retrospect, it appears that the change from Cartesian geometry to spherical geometry was not necessary. This null result, however, also indicates that any 


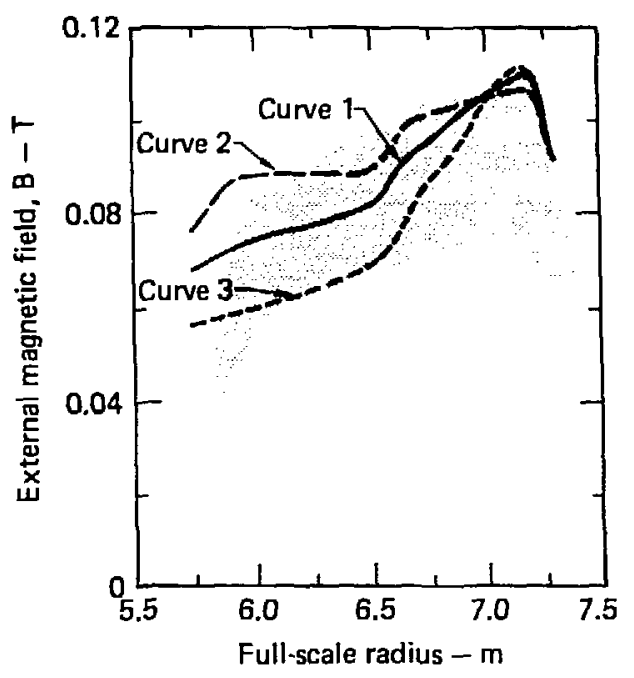

(ncrmalized from 1/10th-scale for measured curves)

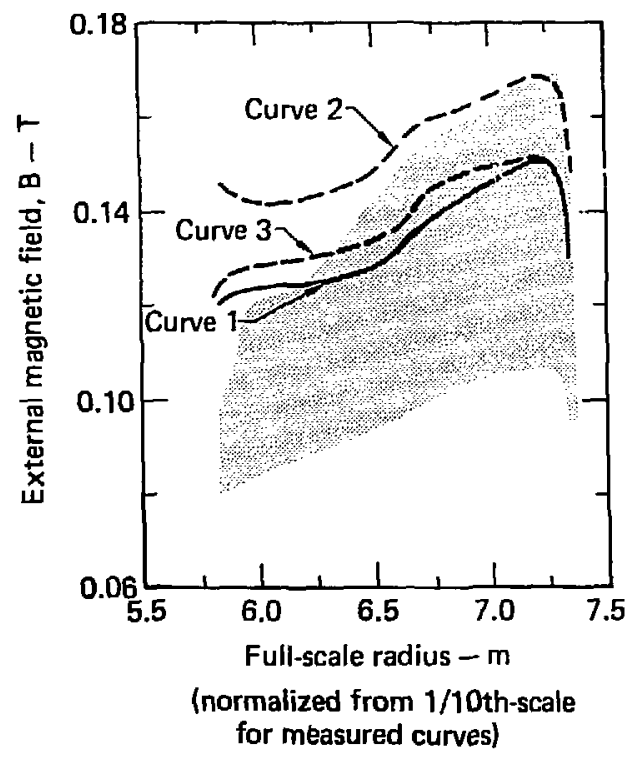

Fig. 21. External magnetic field comparison of MFTF GFUN runs [shaded area from (a) of Fig. 5] with 1/10th-scale model measurements using prototype shield [these are fields between adjacent modules in same tier: Curve 1 = prototype in center of $3 \times 3$ array; Curve 2 = end of one tier (edge of array); Gurve $3=$ corner of array]. Test coils were at $800 \mathrm{~A}$.
Fig. 22. External magnetic field comparison for fields between adjacent modules in adjoining tiers-shaded area from (b) of Fig. 5. 


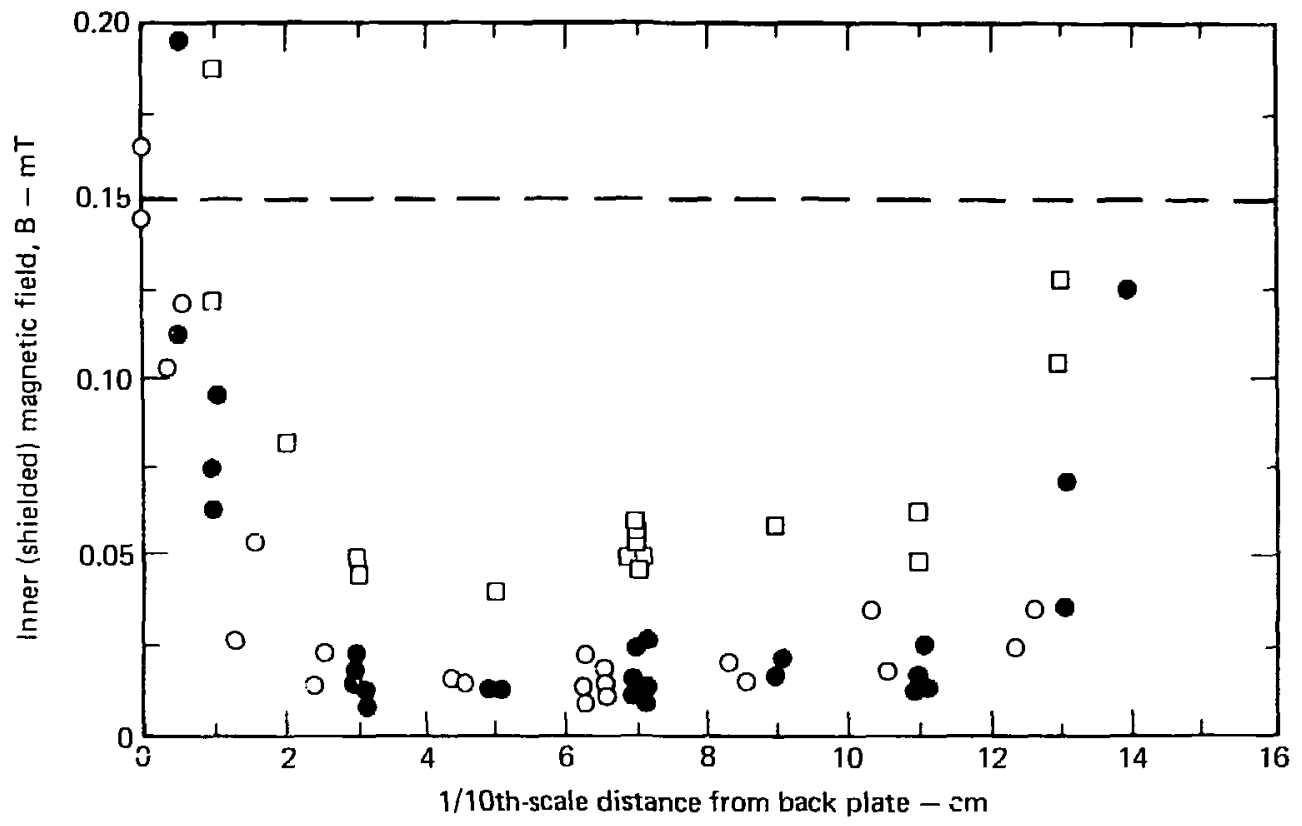

Fig. 23. Inuer magnetic field measurements of prototype: closed circles are for two scans in center of array; open squares are for two edge-position scans; open circles are for two corner-positior scans. Test coils were at $800 \mathrm{~A}$.

remaining differences between the magnetic field environment of the fullscale MFTF coil system and our test environment should not invalidate the results of this prototype test.

As previously discussed, these results also meet the line-integral criterion for the neutralizer. Thus, the prototype passes the test for magnetic shielding.

Figure 24 shows a few measurements that were made of the dc magnetization curve for the prototype test. Note that the inner field starts to rise dramatically when the coil current is raised above the $800-\mathrm{A}$ test level, once again emphasizing that this shield design contains just enough zzgnetic material to perform its function. Saturation effects quickly be$=0=$ dowinant at higher ambient magnetic fields. 


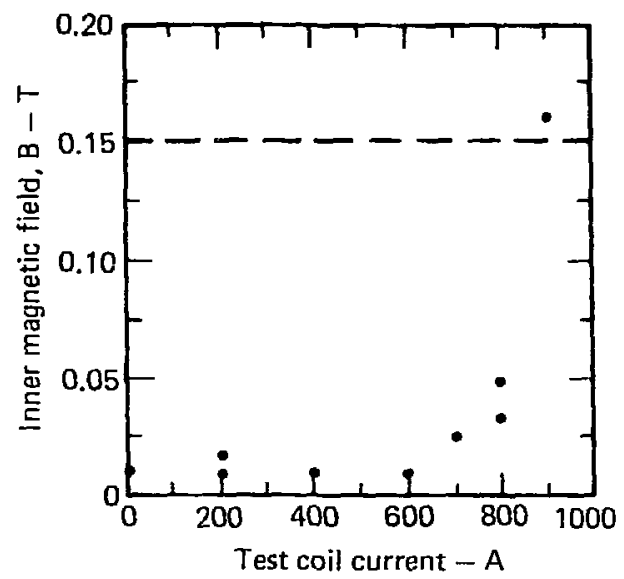

Fig. 24. Direct current magnetization data points for shielded magnetic field in center of prototype at $1 / 10$ th-scale distance of 7 cm from back plate (only a few data points were taken, and roth central and edge positions are plotted together).

No effects were seen in these tests that could be ascribed to magnetic leakage through the gimbal holes. In general, the prototype tests did not produce surprises; they followed the same general trends as the previous measurements .

FULL-SCALE MODULES

Aside from scale, there remain only minor differences between the full-scale shields and the final $1 / 10$ th-scale prototype test. The most noticeable of these is the substitution of Allegheny-Ludlum 4750 nickel-iron alloy for the Carpenter 49 alloy used in the 1/10th-scale measurements. The difference between these materials, however, is not important in the relevant range of magnetic fields. The 0.15-mT upper limit for magnetic field B corresponds to magnetic intensity $H=119 \mathrm{~A}$ turns/m, and inspection of Fig. 7 shows thac the two materials have virtually the same magnetic permeability in this field range. Furthermore, the numerical calculations used the 4750 alloy. 
It must be said that previous magnetic shielding experience was not as useful to us as we originally thought it would be. Most previous fusion experiments used pulsed magnetic fields, and, when that is the case, the skin effect is an important additional aid. Consider the magnetic diffusity $\mathrm{D}_{\mathrm{M}}$ :

$$
\mathrm{D}_{\mathrm{M}}=\frac{\eta}{\mu}=\frac{\delta^{2}}{\tau}
$$

where $n$ is the resistivity of the shield and $\mu$ is its magnetic permeability. From an approximate dimensional analysis, we define the skin depth $\delta$ for a characteristic pulse time $\tau$. For iron below saturation, $\omega_{0} \mu_{0} \geq 10^{3}$ (see Fig. 7), and $\eta=10^{-7} \Omega-m$ at room temperature. In MKS units we obtain:

$$
\delta=0.0089 \sqrt{\tau} \mathrm{m} .
$$

Thus, even at $\tau=1 \mathrm{~s}$, the skin depth $<1 \mathrm{~cm}$, which is indeed a very useful effect, if we have a pulsed field.

In much of the previcus shielding work, there was considerably more space available than in the crowded MFTF module array. Thus, less than optimum designs were often used because it took less design effort to use some of the abundant available space.

Another disappointment was the limitations of the available computer codes. There is no computer code that could accurately simulate the complete three-dimensional array of magnetic shields--or even just one shield. Thus we were forced to build a prototype scale model, which served as an experimental substitute for a three-dimensional analogue -omputer. In this way the basic physica? simplicity of the magnetostatic equations enabled us to solve the problem. One can hope that, in the future, computation techniques will have advanced to the point that recourse to such scaling techniques will no longer be necessary.

\section{ACKNOWLEDGMENTS}

The authors were helped by contributions from R. Bulmer, J. R. Ferguson, B. Johnston, and S. Thomas. This work was part of the Mirror Fusion Test Facility (MFTF) project, under the general direction of $R$. Thomassen and V. Karpenko. 


\section{CONCLUDING REMARKS}

It must be said that previous magnetic shielding experience was not as useful to us as we originally thought it would be. Most previous fusion experiments used pulsed magnetic fields, and, when that is the case, the skin effect is an important additional aid. Consider the magnetic diffusity $\mathrm{D}_{M}$ :

$$
D_{M}=\frac{n}{\mu}=\frac{\delta^{2}}{\tau}
$$

where $n$ is the resistivity of the shield and $\mu$ is its magnetic permeability. From an approximate dimensional analysis, we define the skin depth $\delta$ for a characteristic pulse time $t$. For iron below saturation, $\mu / \mu_{0} \geq 10^{3}$ (see Fig. 7), and $\eta=10^{-7} \Omega-m$ at room temperature. In MKS units we obtain:

$$
\varepsilon=0.0089 \sqrt{\tau} \mathrm{m} .
$$

Thus, even at $\tau=1 \mathrm{~s}$, the skin depth $<1 \mathrm{~cm}$, which is indeed a very useful effect, if we have a pulsed field.

In much of the previcus shielding work, there was considerably more space available than in the crowded MFTF module array. Thus, less than optimum designs were often used because it took less design effort to use some of the abundant available space.

Another disappointment was the limitations of the available computer codes. There is no computer code that could accurately simulate the complete three-dimensional array of magnetic shields--or even just one shield. Thus we were forced to build a prototype scale model, which served as an experimental substitute for a three-dimensional analogue -omputer. In this way the basic physica? simplicity of the magnetostatic equations enabled us to solve the problem. One can hope that, in the future, computation techniques will have advanced to the point that recourse to such scaling techniques will no longer be necessary.

\section{ACKNOWLEDGMENTS}

The authors were helped by contributions from R. Bulmer, J. R. Ferguson, B. Johnston, and S. Thomas. This work was part of the Mirror Fusion Test Facility (MFTF) project, under the general direction of R. Thomassen and V. Karpenko. 


\section{REFERENCES}

1. The Mirror Fusion Test Facility, Lawrence Livermore Laboratories, Livermore, CA, Report LLL-TB-009 (1978).

2. K. Thomassen, Lawrence Livermore Laboratory, Internal Document MFE/CP/78-334 (1978). Readers outside the Laboratory who desire further information on LLL internal documents should address their inquiries to the Technical Information Department, Lawrence Livermore Laboratory, Livermore, CA 94550.

3. A. W. Molvik, Lawrence Livermore Laboratory, Internal Document MFE/CP/77-186 (1977).

4. J. W. Shearer, Lawrence Livermore Laboratory, Internal Document $\mathrm{MFE} / \mathrm{CP} / 78-161$ (1978).

5. A. G. Armstrong, C. J. Collis, N. J. Diserens, M. J. Newman, J. Simkin, and C. W. Trowbridge, GFUN3D User Guide, Rutherford Laboratory (Oxon, OX11, OQX, England, Report RL-76-029/A (1976).

6. J. H. Foote, Lawrence Livermore Laboratory, Interna1 Document MFE/CP/79-33 (1979).

7. J. S. Colonias, Particle Accelerator Design: Computer Programs, (Academic Press, New York, 1974).

8. S. J. Sackett, EFFI-A Code for Calculating the Electromagnetic Field, Force, and Inductance in Coil Sygtems of Arbitrary Geometry, Lawrence Livermore Laboratory, Livermore, CA, UCRL-52402 (1978).

9. A. W. Molvik, "Neutral Beam Sources," in Magnetic Fusion Energy Quarterly Report, October through December 1978, M. A. Harrison and C. K. McGregor, Eds., Lawrence Livermore Laboratory, Livermore, CA, UCRL-50051-78-4 (1979), p. 27. 